\title{
Results and prospects with the CMS-TOTEM Precision Proton Spectrometer
}

\author{
Laurent Forthomme ${ }^{* \dagger}$ \\ Helsinki Institute of Physics, University of Helsinki, Finland \\ E-mail: laurent.forthommedcern.ch
}

\begin{abstract}
With more than $100 \mathrm{fb}^{-1}$ recorded during LHC run 2 (2016-2018), the joint CMS-TOTEM Precision Proton Spectrometer (CT-PPS) has started to deliver its first physics results. Located between 200 and $220 \mathrm{~m}$ on each side from the CMS detector, it aims at detecting forward scattered protons emerging intact from the interaction, as for instance in central exclusive processes (CEP). In this paper we describe in detail the search for two-photon production of a lepton pair performed with the first $10 \mathrm{fb}^{-1}$. This result proves the feasibility of operating near-beam detectors at the LHC under standard, high luminosity conditions. In addition, we describe the potential of PPS for future physics studies.
\end{abstract}

European Physical Society Conference on High Energy Physics - EPS-HEP2019 -

10-17 July, 2019

Ghent, Belgium

\footnotetext{
* Speaker.

${ }^{\dagger}$ on behalf of the CMS and TOTEM Collaborations.
} 


\section{CMS-TOTEM Precision Proton Spectrometer}

Located between 200 and 220 metres away from the CMS [1] interaction point, CT-PPS (since 2018 referred to as PPS) [2] allows to detect forward scattered protons in standard, high-luminosity LHC collision. As pictured in Figure 1, an array of tracking and timing detectors is installed close to the beam, and inserted at a $15 \sigma$ distance of approach (in units of standard deviations of the beam transverse distribution) under stable beams conditions.

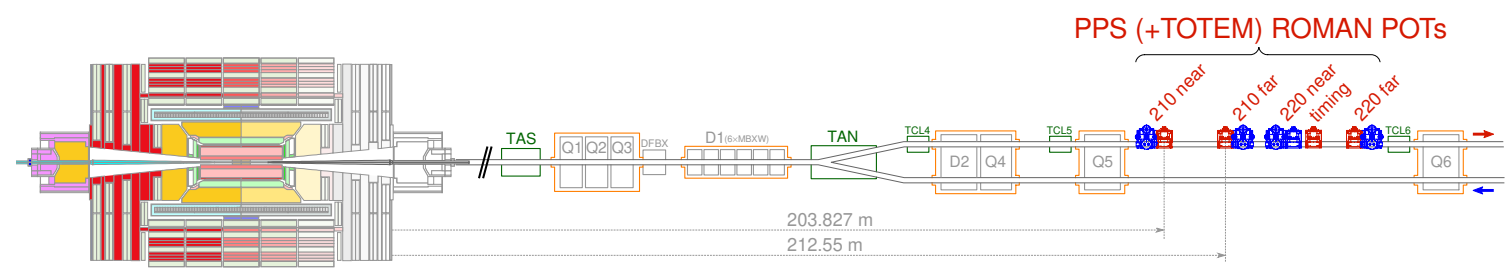

Figure 1: A schematic layout of one of the two arms of the Precision Proton Spectrometer within the CMS environment. In red and blue are depicted (resp.) the horizontal PPS and the vertical TOTEM Roman pots inserted close to the LHC beams lines.

Tracking detectors are used in the reconstruction of the horizontal and vertical displacement of protons path with respect to the beam, after their interaction in the central part of CMS. Given the optical properties of the beamline elements between the central detector and CT-PPS detectors (housed in Roman Pots, RPs), the (horizontal) displacement is directly linked to the proton momentum loss (or its reduced form $\xi=\Delta p / p$ ). Additionally, timing detectors are measuring the time of arrival of each of these protons. For double-arm measurements this allows for the reconstruction of the longitudinal position of the interaction, and hence a good events pileup mitigation. Over the full (CT-)PPS operations in LHC run 2, four technologies were used in the two components, each along different periods.

The tracking detectors consisted of two stations containing ten planes of TOTEM edgeless silicon strip sensors (2016-2017), gradually replaced by two stations of six planes of 3D pixels (2017-2018). The main reasons for this change of technology are an improved radiation tolerance compatible with the dose accumulated over a LHC running period at high luminosity, and the added feature to reconstruct events with multiple forward scattered tracks.

For timing detectors, one station of single-crystal CVD diamond sensors was installed in each arm from the second part of 2016 on. During the 2017 data collection, the last plane of diamond was replaced by an ultra-fast silicon LGAD sensor.

A second arm with same detector types is installed in the opposite LHC sector, thus making the spectrometer fully symmetric with respect to the central detector.

The RPs being retracted between each LHC fill, and inserted once beams reach stability, a dedicated alignment technique is used to allow the reconstruction of the tracks impact point with respect to the beam distribution. A specific low-intensity fill is used, in which the pots are inserted at a distance of $5 \sigma$ from the beam. The first beam-based alignment uses the collimators to scrape the beam, thus generating a characteristic signal in the beam loss monitors. Additionally, the 
relative per-pots alignment (translation and rotations) exploits the hit and track correlations among the stations. The absolute alignment is then obtained from the observation of the beam position from elastic $p p \rightarrow p p$ scattering events with vertical pots. The last iteration relies on the matching of a geometrical distribution (for instance, the horizontal component of tracks impact point) to align each high-luminosity fill to the reference run obtained in previous steps. A more detailed description of the full alignment procedure may be found in [3].

As further discussed in [4], the connection between the reconstructed tracks impact point properties and the proton kinematics may be expressed as a linearised beamline transfer function. For a given state vector $\mathbf{d}^{*}$ expressed at the interaction point, the state at a given $s$ coordinate reads $\mathbf{d}(s)=T(s, \boldsymbol{\xi}) \mathbf{d}^{*}$. In this formalism, the single-pass transfer matrix $T(s, \xi)$ is calculated from the simulation of intermediate optical elements responses, and constrained from RPs observations. The forward tracks observables encompassed in a state vector are the transverse positions $x^{(*)}, y^{(*)}$ and angles $\theta_{x}^{(*)}, \theta_{y}^{(*)}$, along with the relative proton momentum loss $\xi$.

\section{Observation of $\gamma \gamma \rightarrow l^{+} l^{-}$with forward protons}

Using the alignment and beamline optics determination briefly described above, a search for two-photon production of a pair of leptons (muons or electrons) has been performed using $9.4 \mathrm{fb}^{-1}$ collected in 2016 at a centre-of-mass energy $\sqrt{s}=13 \mathrm{TeV}$ [5]. This corresponds to a period when only CT-PPS horizontal strips were inserted, thus with no timing information.
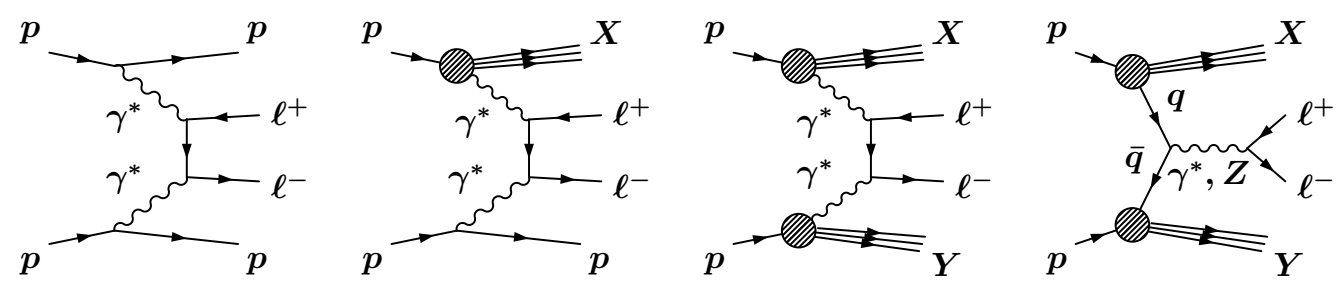

Figure 2: From left to right: central exclusive, semi-exclusive (not depicting the symmetric diagram), and dissociative two-photon production of a lepton pair, and dominant inclusive Drell-Yan background contribution.

As pictured in the first three diagrams of Figure 2, the photon emission from protons may result from an elastic or dissociative process, primarily depending on the fractional momentum transfer involved in the exchange. This results in four different topologies, where either both, one, or neither the protons remain undissociated after the photon emission, and may thus be detected if within the RPs acceptance.

The cross section for the fully elastic component peaks at low $\xi$ values (as predicted in e.g. LPAIR [6]) and quickly decreases at higher invariant masses. In this scheme, the scattered protons momentum loss may be connected to the central system kinematics through:

$$
m_{\text {c.m. }}=\sqrt{s \xi_{1} \xi_{2}}, \quad y_{\text {c.m. }}=\frac{1}{2} \log \left(\frac{\xi_{1}}{\xi_{2}}\right)
$$

The strategy adopted in this search was hence to increase the statistics available by allowing a single proton tag. The proton kinematics measured with CT-PPS was compared with that derived 
from the dilepton pair, estimated as:

$$
\xi^{ \pm}\left(l^{+} l^{-}\right)=\frac{1}{\sqrt{s}}\left(p_{\mathrm{T}}\left(l^{+}\right) e^{ \pm \eta\left(l^{+}\right)}+p_{\mathrm{T}}\left(l^{-}\right) e^{ \pm \eta\left(l^{-}\right)}\right),
$$

where the positive (resp. negative) superscript refers to a proton scattering towards the LHC sector 45 (resp. 56), according to the CMS convention.

In the central detectors, a $\gamma \gamma$ fusion-enhanced kinematic selection is defined (following the previous CMS and ATLAS run 1 and 2 searches for $\gamma \gamma$ production processes) through a tight cut on the difference of azimuthal angle between the two leptons, or acoplanarity $1-|\Delta \phi / \pi|$, and a veto on additional tracks reconstructed in the vicinity of the dilepton vertex. In the dielectron and dimuon channels, the acoplanarity is required to be less than 0.6 and $0.9 \%$, respectively. As for the tracks veto size, it is required that no tracks are produced within a radius of $0.5 \mathrm{~mm}$ around the refitted dilepton vertex, for both the final states.

The main sources of background include inclusive Drell-Yan (though a large suppression is predicted from the $m\left(l^{+} l^{-}\right)>110 \mathrm{GeV}$ selection) and double-dissociative two-photon production, overlaid with a pileup proton from a different interaction. These two sources are shown in the fourth and third diagrams of Figure 2, respectively. To evaluate the probability for such sources to pass the central and forward selections for the earlier, a sample of $Z \rightarrow l^{+} l^{-}$events is selected and mixed with a randomised collection of proton tracks extracted from data. For the double-dissociative contribution, a similar technique is using a realistic central kinematics simulated with LPAIR, and scaled to its theoretical cross section. The expected background yields for the electron (resp. muon) channel within the full CT-PPS longitudinal momentum loss acceptance is $10.5 \pm 2.1$ (resp. $11.0 \pm 4.0$ ) events. A total of $2.36 \pm 0.09$ (stat) \pm 0.47 (syst) and $1.49 \pm 0.07$ (stat) \pm 0.53 (syst) background events are expected in the dielectron and dimuon channels, respectively.

As shown in Figure 3, a total of $8 e^{+} e^{-}$and $12 \mu^{+} \mu^{-}$candidates are observed in $9.4 \mathrm{fb}^{-1}$ collected at $13 \mathrm{TeV}$ in 2016 . This corresponds to a significance of $2.6 \sigma$ and $4.3 \sigma$ respectively over the background predictions. No events were observed with protons reconstructed in both arms, consistent with theoretical calculations in this kinematic range.

The two lepton channels may be combined yielding an overall significance of $5.1 \sigma$, thus establishing the first observation of the $p p \rightarrow p^{(*)}\left(\gamma \gamma \rightarrow l^{+} l^{-}\right) p$ process with proton tagging. With the highest mass dielectron event of $917 \mathrm{GeV}$, the electroweak scale is reached for such a $\gamma \gamma$ fusion process.

\section{PPS prospects with $100 \mathrm{fb}^{-1}$}

With the first result described above, the CT-PPS detectors have demonstrated their capability to operate on a regular basis under demanding, high-luminosity conditions. A gradual upgrade of detectors and their technologies has been performed since 2016 and first operations of CT-PPS, both for tracking and timing detectors.

This makes it possible to explore a number of processes already with the $100 \mathrm{fb}^{-1}$ collected during LHC run 2. Among these, new particles exchanges, as well as effective field theory extensions of the Standard model. One may quote, among other final states, the two-photon production of 

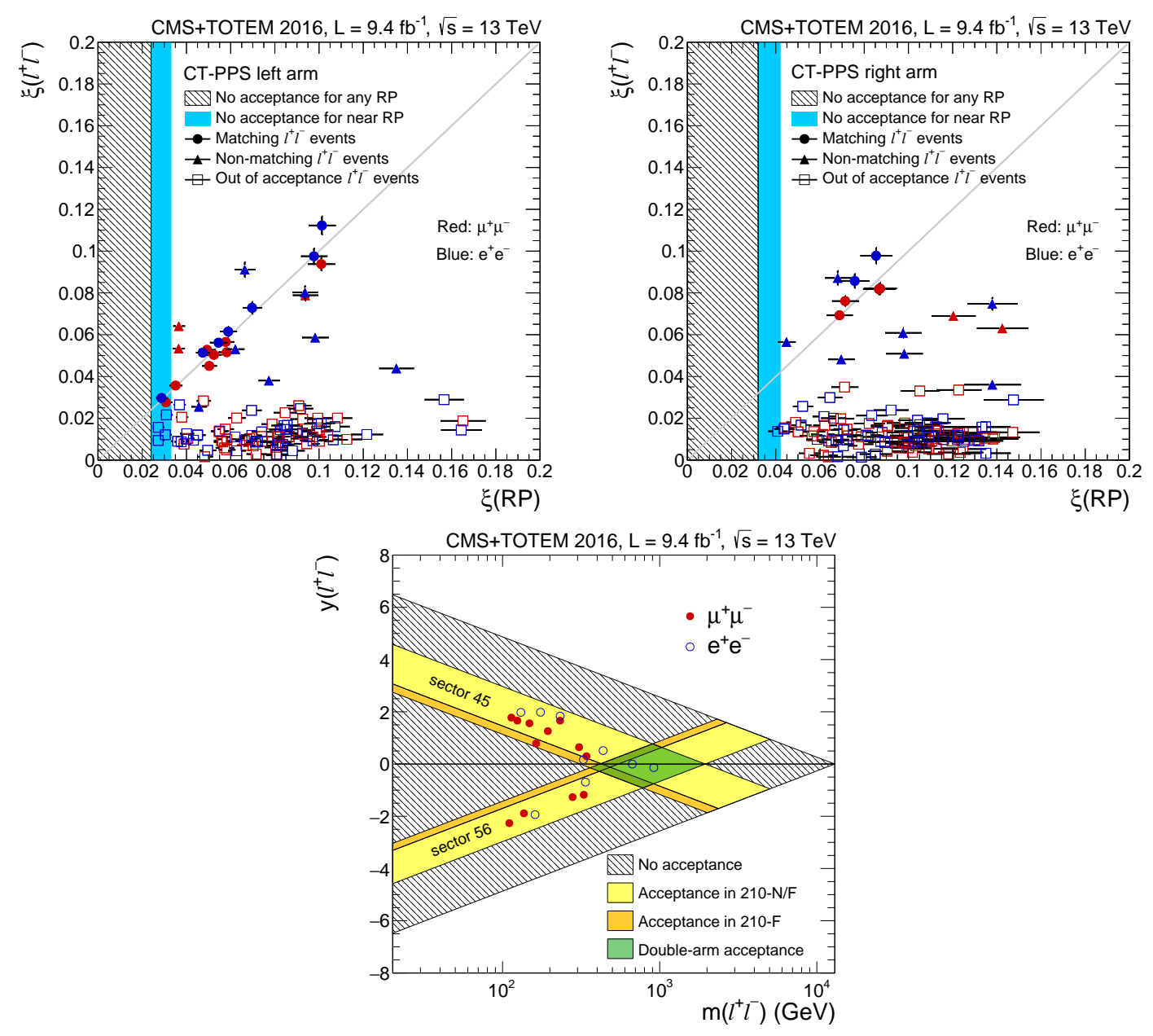

Figure 3: Top row: Correlations between relative momentum losses $\xi$ as reconstructed from the PPS tracking detectors information and the central dilepton system, for the LHC sectors 45 (left) and 56 (right). Bottom figure: Distribution of dimuon (red) and dielectron (blue) candidates with matching $\xi$ values observed in the dilepton mass/rapidity plane. The hatched regions correspond to kinematics ranges outside both RPs acceptance within one spectrometer arm. All figures are extracted from [5].

$\gamma \gamma$ (for instance, considering axion-like particles exchanges or anomalous quartic gauge couplings), $W^{+} W^{-}$(with a two orders of magnitude improvement in sensitivity compared to analyses without proton detection, see e.g. [2]), ZZ, or supersymmetric partners of leptons and gauge bosons.

\section{Summary}

The CMS-TOTEM Precision Proton Spectrometer has been successfully integrated in the CMS experiment since 2016, and has collected over $100 \mathrm{fb}^{-1}$ of data at $13 \mathrm{TeV}$ during LHC run 2. It has demonstrated its capability to operate under standard LHC luminosity conditions on a regular basis, and has allowed to observe for the first time the two-photon production of a lepton pair with proton tagging, with candidates reaching the electroweak scale. 


\section{References}

[1] S. Chatrchyan et al. [CMS Collaboration], JINST 3 (2008) S08004, doi:10.1088/1748-0221/3/08/S08004.

[2] M. Albrow et al. [CMS and TOTEM Collaborations], CERN-LHCC-2014-021, TOTEM-TDR-003, CMS-TDR-13.

[3] J. Kašpar, CERN-TOTEM-NOTE-2017-001.

[4] F. Nemes, CERN-TOTEM-NOTE-2017-002.

[5] A. M. Sirunyan et al. [CMS and TOTEM Collaborations], JHEP 1807 (2018) 153 doi:10.1007/JHEP07(2018)153 [arXiv:1803.04496 [hep-ex]].

[6] S. P. Baranov, O. Duenger, H. Shooshtari and J. A. M. Vermaseren, Hamburg 1991, Proceedings, Physics at HERA, vol. 31478. 